DESY 17-161, CERN-TH-2017-204

Prepared for: Multiple Parton Interactions at the LHC

Eds. P. Bartalini and J. R. Gaunt, World Scientific, Singapore

\title{
Double Parton Scattering - Theory Overview
}

\author{
Markus Diehl* and Jonathan R. Gaunt ${ }^{\dagger}$ \\ * Deutsches Elektronen-Synchroton DESY, \\ Notkestraße 85, 22607 Hamburg, Germany \\ ${ }^{\dagger}$ CERN Theory Division, \\ 1211 Geneva 23, Switzerland
}

\begin{abstract}
The dynamics of double hard scattering in proton-proton collisions is quite involved compared with the familiar case of single hard scattering. In this contribution, we review our theoretical understanding of double hard scattering and of its interplay with other reaction mechanisms.
\end{abstract}

\section{Introduction}

The most familiar mechanism for hard processes in proton-proton collisions is single parton scattering (SPS): two partons, one from each proton, undergo a hard scattering that produces heavy particles or particles with high transverse momenta. For the cross section one then has a factorisation formula, containing a parton distribution function (PDF) for each proton and a parton-level cross section for the hard subprocess. Double parton scattering (DPS) occurs if in the same proton-proton collision two partons in each proton initiate two separate hard scattering processes. The corresponding factorisation formula contains two parton-level cross sections and a double parton distribution (DPD) for each proton. The two hard scatters are separated by a finite distance $\boldsymbol{y}$ in the plane transverse to the colliding proton momenta, so that a DPD depends not only on the momentum fractions $x_{1}$ and $x_{2}$ of two partons, but also on the transverse distance $\boldsymbol{y}$ between them. Very roughly, DPDs should grow like the square of two ordinary PDFs when $x_{1}$ and $x_{2}$ become small. The importance of DPS compared with SPS is hence increased in this small $x$ region, which for a given final state becomes more and more important with growing collision energy. 
The single and double parton distributions just described are integrated over transverse parton momenta; they are often called "collinear" distributions, and the associated formalism is called "collinear factorisation". The information on transverse parton momenta is retained in so-called TMDs (transverse momentum dependent distributions). The corresponding TMD factorisation formulae allow one to compute cross sections differential in the transverse momentum $\boldsymbol{q}$ of a heavy particle (e.g. a $Z$ or a Higgs boson) in the region where $\boldsymbol{q}$ is much smaller than the boson mass. The TMD concept can be extended to DPS processes, for instance to describe the region of low transverse boson momenta $\boldsymbol{q}_{1}$ and $\boldsymbol{q}_{2}$ in $W^{+} W^{+}$or $H Z$ production. This is especially valuable because the importance of DPS compared with SPS is much higher in the cross section for measured small $\boldsymbol{q}_{1}$ and $\boldsymbol{q}_{2}$ than it is in the integrated cross section.

Factorisation for SPS processes has been derived within QCD to a high level of rigour, as reviewed for instance in Ref. 1. It is an ongoing effort to bring factorisation for DPS to a comparable standard. In the present contribution, we review the status of this effort. Note that we are discussing so-called "hard scattering factorisation" here, which is based on separating dynamics at different distance scales. This is distinct from "high-energy" or "small $x$ factorisation", where the separation criterion is rapidity. Some discussion of this concept in the context of DPS is given in Ref. 2.

\section{Cross section formula}

Let us start with a main theory result: the cross section formula for DPS. Consider the production of two particles with invariant masses $Q_{1}, Q_{2}$ and transverse momenta $\boldsymbol{q}_{1}, \boldsymbol{q}_{2}$. We require that $Q_{1}$ and $Q_{2}$ be large and generically denote their size by $Q$. Instead of a heavy particle, one may also have a system of particles with large invariant mass, for instance a dijet.

Collinear factorisation allows us to compute the cross section integrated over $\boldsymbol{q}_{1}$ and $\boldsymbol{q}_{2}$ :

$$
\begin{aligned}
& \frac{d \sigma_{\mathrm{DPS}}}{d x_{1} d x_{2} d \bar{x}_{1} d \bar{x}_{2}}=\frac{1}{C} \sum_{a_{1} a_{2} b_{1} b_{2}} \int_{x_{1}}^{1-x_{2}} \frac{d x_{1}^{\prime}}{x_{1}^{\prime}} \int_{x_{2}}^{1-x_{1}^{\prime}} \frac{d x_{2}^{\prime}}{x_{2}^{\prime}} \int_{\bar{x}_{1}}^{1-\bar{x}_{2}} \frac{d \bar{x}_{1}^{\prime}}{\bar{x}_{1}^{\prime}} \int_{\bar{x}_{2}}^{1-\bar{x}_{1}^{\prime}} \frac{d \bar{x}_{2}^{\prime}}{\bar{x}_{2}^{\prime}} \\
& \quad \times \sum_{R}{ }^{R} \hat{\sigma}_{a_{1} b_{1}}\left(x_{1}^{\prime} \bar{x}_{1}^{\prime} s, \mu_{1}^{2}\right)^{R} \hat{\sigma}_{a_{2} b_{2}}\left(x_{2}^{\prime} \bar{x}_{2}^{\prime} s, \mu_{2}^{2}\right) \\
& \quad \times \int d^{2} \boldsymbol{y} \Phi^{2}(y \nu)^{R} F_{b_{1} b_{2}}\left(\bar{x}_{i}^{\prime}, \boldsymbol{y} ; \mu_{i}, \bar{\zeta}\right)^{R} F_{a_{1} a_{2}}\left(x_{i}^{\prime}, \boldsymbol{y} ; \mu_{i}, \zeta\right)
\end{aligned}
$$


Note that we use boldface for any vector $\boldsymbol{w}$ in the transverse plane and denote its length by $w=|\boldsymbol{w}|$. There are strong indications ${ }^{33}$ that TMD factorisation in SPS works only for the production of colourless particles, so that we make the same restriction for DPS. The differential cross section for transverse momenta $\left|\boldsymbol{q}_{1}\right|,\left|\boldsymbol{q}_{2}\right| \sim q_{T}$ much smaller than $Q$ reads

$$
\begin{aligned}
& \frac{d \sigma_{\mathrm{DPS}}}{d x_{1} d x_{2} d \bar{x}_{1} d \bar{x}_{2} d^{2} \boldsymbol{q}_{1} d^{2} \boldsymbol{q}_{2}}=\frac{1}{C} \sum_{a_{1} a_{2} b_{1} b_{2}} \hat{\sigma}_{a_{1} b_{1}}\left(Q_{1}^{2}, \mu_{1}^{2}\right) \hat{\sigma}_{a_{2} b_{2}}\left(Q_{2}^{2}, \mu_{2}^{2}\right) \\
& \times \int d^{2} \boldsymbol{y} \frac{d^{2} \boldsymbol{z}_{1}}{(2 \pi)^{2}} \frac{d^{2} \boldsymbol{z}_{2}}{(2 \pi)^{2}} e^{-i\left(\boldsymbol{q}_{1} \boldsymbol{z}_{1}+\boldsymbol{q}_{2} \boldsymbol{z}_{2}\right)} \Phi\left(y_{+} \nu\right) \Phi\left(y_{-} \nu\right) \\
& \times \sum_{R}{ }^{R} F_{b_{1} b_{2}}\left(\bar{x}_{i}, \boldsymbol{z}_{i}, \boldsymbol{y} ; \mu_{i}, \bar{\zeta}\right)^{R} F_{a_{1} a_{2}}\left(x_{i}, \boldsymbol{z}_{i}, \boldsymbol{y} ; \mu_{i}, \zeta\right)
\end{aligned}
$$

These formulae are quite complex. In the following we briefly explain their different ingredients, and the physics behind them.

We begin with the simplest ones. The variables $x_{i}$ and $\bar{x}_{i}$ are given by

$$
x_{i}=Q_{i} e^{Y_{i}} / \sqrt{s}, \quad \bar{x}_{i}=Q_{i} e^{-Y_{i}} / \sqrt{s}, \quad(i=1,2)
$$

where $Y_{i}$ is the centre-of-mass rapidity of the system $i$ and $\sqrt{s}$ the overall collision energy. $C$ is a combinatorial factor, equal to 2 if the systems 1 and 2 are identical, and equal to 1 otherwise.

The parton-level cross sections $\hat{\sigma}$ are precisely the same as the ones in the corresponding SPS cross sections, except for the superscript $R$ in (1), which will be explained below. They include the effects of hard QCD radiation in the process. In TMD factorisation, $\hat{\sigma}$ receives only virtual corrections, since hard real radiation tends to knock $\boldsymbol{q}_{i}$ out of the region $q_{T} \ll Q_{i}$. As a consequence, the momentum fractions of the partons entering the hard subprocesses are fixed to $x_{i}$ and $\bar{x}_{i}$ by external kinematics. In collinear factorisation, $\hat{\sigma}$ includes real emission, which allows for momentum fractions $x_{i}^{\prime} \geq x_{i}$ and $\bar{x}_{i}^{\prime} \geq \bar{x}_{i}$.

The joint distribution of two partons in a proton is quantified by the DPDs $F$, which have two labels $a_{i}$ for the parton type, two momentum fraction arguments $x_{i}$, and two factorisation scales $\mu_{i}$ (they can be chosen separately, which is useful if $Q_{1}$ and $Q_{2}$ are of different size). In the TMD case there are two transverse position arguments $\boldsymbol{z}_{i}$, which are Fourier conjugate to the transverse parton momenta $\boldsymbol{k}_{i}$. The structure $\int d^{2} \boldsymbol{z}_{i} e^{-i \boldsymbol{q}_{i} \boldsymbol{z}_{i}} F\left(\bar{x}_{i}, \boldsymbol{z}_{i}, \cdots\right) F\left(x_{i}, \boldsymbol{z}_{i}, \cdots\right)$ in (2) is the same as in the corresponding factorisation formula for SPS - in momentum space it corresponds to a convolution product $\int d^{2} \boldsymbol{k}_{i} F\left(\bar{x}_{i}, \boldsymbol{q}_{i}-\boldsymbol{k}_{i}, \cdots\right) F\left(x_{i}, \boldsymbol{k}_{i}, \cdots\right)$. 
As already mentioned, a DPD also depends on the distance $\boldsymbol{y}$, which in collinear factorisation literally corresponds to the transverse distance between the two active partons in the proton, and thus to the distance between the two hard-scattering processes. In the TMD case, $\boldsymbol{y}$ corresponds to the average distance between the partons in the scattering amplitude and its conjugate, as can be seen in (4) and (5) below. Notice that in the cross section, $\boldsymbol{y}$ is not Fourier conjugate to any observable momentum, unlike $\boldsymbol{z}_{i}$.

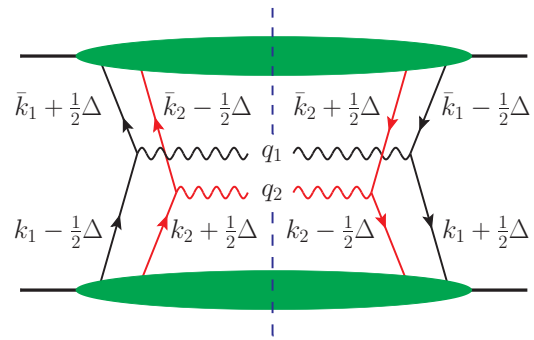

(a)

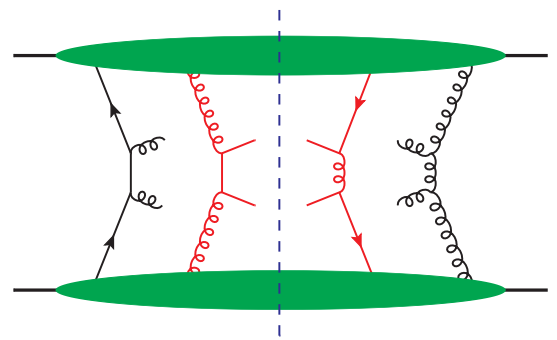

(b)

Fig. 1. (a) Tree level graph for the production of two electroweak gauge bosons by DPS (often called double Drell-Yan). The blobs represent DPDs. The graph is for the cross section, with the vertical line indicating the final state cut. (b) Graph for double dijet production with DPDs for quark-gluon interference.

It is instructive to see how the distance $\boldsymbol{y}$ emerges from the analysis of Feynman graphs in momentum space. The longitudinal momentum fractions of each parton are fixed by the final state kinematics and thus must be equal in the scattering amplitude and its conjugate. By contrast, the transverse parton momenta can differ by an amount $\boldsymbol{\Delta}$ or $-\boldsymbol{\Delta}$ as shown in Fig. 1(a). The momentum mismatch for the first and the second parton is opposite in sign, so that the transverse momentum of the spectator partons is the same in the amplitude and its conjugate. Since this momentum mismatch is not observable, one has an integral of the form $\int d^{2} \boldsymbol{\Delta} F\left(\bar{x}_{i},-\boldsymbol{\Delta}, \cdots\right) F\left(x_{i}, \boldsymbol{\Delta}, \cdots\right)$ in the cross section. A Fourier transform from $\boldsymbol{\Delta}$ to $\boldsymbol{y}$ gives the form shown in (1) and (2). More detail on the tree-level derivation of the factorised structure in collinear factorisation is given in Ref. 4 .

Let us now turn to the quantum numbers of the partons. Even in an unpolarised proton, two extracted partons can have correlations between their polarisations. The labels $a_{i}, b_{i}$ in the cross section formulae refer not 
only to the type of the parton but also to its polarisation, and one must sum over all allowed combinations. An example for a polarisation dependent DPD is $F_{\Delta q \Delta q}$, which corresponds to the difference of distributions for two quarks with equal helicities and for two quarks with opposite helicities.

Not only the transverse momentum of a parton can differ between the amplitude and its conjugate, but also its colour. The different possible colour combinations in DPDs and the parton-level cross sections are specified by the label $R$. For the production of colourless particles there is only one possible colour structure for $\hat{\sigma}$, which hence requires no index $R$ in the TMD formula (2). Let us explain the meaning of $R$ for the simplified setting of the tree graph in Fig. 1(a). In each DPD, one can couple the two parton lines with momentum fraction $x_{1}\left(\bar{x}_{1}\right)$ to be in the colour representation $R=1,8, \ldots$ (the two other lines then are in the conjugate representation, because all four lines must couple to an overall singlet). In colour singlet distributions ${ }^{1} F$, partons with equal momentum fractions thus have equal colour - this is the only possible combination for single parton distributions. Colour non-singlet DPDs describe colour correlations. At the end of Sec. 6 we will see that they are suppressed by Sudakov logarithms if the scale of the hard process is large.

Finally, there also exist DPDs describing the interference between different parton types in the amplitude and its conjugate, be it between different quark flavours, between quarks and antiquarks, or between quarks and gluons. For ease of notation, they are not included in the cross section formulae (11) and (2). An example for quark-gluon interference in double dijet production is given in Fig. 1(b). Parton type interference distributions do not have any dynamical cross talk with gluon DPDs, which have the strongest enhancement at small $x_{i}$. In many situations, one can therefore expect them to play only a minor role. A detailed discussion of correlations in DPDs can be found in Ref. 5 .

DPDs can be defined via operator matrix elements, which provides a solid field theoretical basis for their investigation. For a double quark TMD one writes

$$
\begin{gathered}
{ }^{R} F_{a_{1} a_{2}}\left(x_{i}, \boldsymbol{z}_{i}, \boldsymbol{y} ; \mu_{i}, \zeta\right)=2 p^{+} \int d y^{-} \frac{d z_{1}^{-}}{2 \pi} \frac{d z_{2}^{-}}{2 \pi} e^{i\left(x_{1} z_{1}^{-}+x_{2} z_{2}^{-}\right) p^{+}} \\
\times\left\langle p\left|\mathcal{O}_{a_{2}}\left(0, z_{2}\right) \mathcal{O}_{a_{1}}\left(y, z_{1}\right)\right| p\right\rangle \times\{\text { soft factor }\}
\end{gathered}
$$

where we use light-cone coordinates $w^{ \pm}=\left(w^{0} \pm w^{3}\right) / \sqrt{2}$ for any four-vector $w^{\mu}$. It is understood that $\boldsymbol{p}=\mathbf{0}$ and that the proton spin is averaged over. The bilinear operators $\mathcal{O}$ are the same as in the definition of a single parton 
TMD. They are given by

$$
\mathcal{O}_{a}(y, z)=\left.\bar{q}\left(y-\frac{1}{2} z\right) W^{\dagger}\left(y-\frac{1}{2} z\right) \Gamma_{a} W\left(y+\frac{1}{2} z\right) q\left(y+\frac{1}{2} z\right)\right|_{z^{+}=y^{+}=0}
$$

with a past-pointing light-like Wilson line

$$
W(\xi)=\mathrm{P} \exp \left[i g t^{a} \int_{0}^{\infty} d s n A^{a}(\xi-s n)\right],
$$

where $\mathrm{P}$ denotes path-ordering and $n$ is a light-like vector $\left(n^{-}=1, n^{+}=0\right.$, $\boldsymbol{n}=\mathbf{0})$. The dynamical origin of this Wilson line is explained in Sec. 6 $\Gamma_{a}$ is a Dirac matrix and determines the quark polarisation. In particular, unpolarised quarks correspond to $\Gamma_{q}=\frac{1}{2} \gamma^{+}$, and longitudinal quark polarisation is described by $\Gamma_{\Delta q}=\frac{1}{2} \gamma^{+} \gamma_{5}$.

The "soft factor" in (4) originates from soft gluon exchange in the physical scattering process and gives rise to the dependence on a parameter $\zeta$, as explained in Sec. 6. Such a dependence is already present in single parton TMDs. Moreover, the operator (5) and the soft factor contain ultraviolet divergences, which require renormalisation. This brings in the dependence on the renormalisation scales $\mu_{i}$. Finally, the dependence of the DPD on $R$ arises from the colour indices of the operators $\left(\bar{q} W^{\dagger}\right)_{i^{\prime}}$ and $(W q)_{i}$ in (5) and from the soft factor. Again, more detail is given in Sec. 6.

The preceding discussion can be repeated for antiquarks or gluons, with different operators $\mathcal{O}_{a}$. The definition of collinear DPDs $F_{a_{1} a_{2}}\left(x_{i}, \boldsymbol{y} ; \mu_{i}, \zeta\right)$ reads as in (4) but with $\boldsymbol{z}_{i}=\mathbf{0}$. Note that in the colour non-singlet case, the soft factor and the dependence on $\zeta$ do not drop out in the collinear case. Putting $\boldsymbol{z}_{i}$ to zero introduces additional ultraviolet divergences, so that the renormalisation and hence the $\mu_{i}$ dependence is quite different between TMDs and collinear distributions, as we will see later.

The role of the function $\Phi$ in (11) and (2) will be explained in Sec. 4. It is closely related to the fact that the cross section of a physical process receives not only contributions from DPS, but also from SPS and possibly other mechanisms. In the next section, we give an overview of these.

\section{Power behaviour}

The factorisation of cross sections into perturbative hard-scattering subprocesses and nonperturbative quantities like parton distributions is based on an expansion in the small parameter $\Lambda / Q$. Here $Q$ denotes the scale of the hard scattering and $\Lambda$ a typical hadronic scale. For simplicity, we treat the size of the transverse momenta $\boldsymbol{q}_{1}$ and $\boldsymbol{q}_{2}$ in TMD factorisation 
as order $\Lambda$ here. The case where they are much larger than a hadronic scale (but still much smaller than $Q$ ) is discussed in Sec. 8 .

Dimensional analysis of the TMD factorisation formulae for SPS and DPS reveals that the two mechanisms have the same power behaviour:

$$
\frac{d \sigma_{\mathrm{SPS}}}{d^{2} \boldsymbol{q}_{1} d^{2} \boldsymbol{q}_{2}} \sim \frac{d \sigma_{\mathrm{DPS}}}{d^{2} \boldsymbol{q}_{1} d^{2} \boldsymbol{q}_{2}} \sim \frac{1}{\Lambda^{2} Q^{4}} .
$$

The situation changes if one integrates over $\boldsymbol{q}_{1}$ and $\boldsymbol{q}_{2}$. In DPS both are of order $\Lambda$ since they originate from the transverse momenta of partons inside the colliding protons. In SPS this holds only for the sum $\boldsymbol{q}_{1}+\boldsymbol{q}_{2}$, whilst the individual momenta $\boldsymbol{q}_{1}$ and $\boldsymbol{q}_{2}$ (and thus their difference) are only limited by the available phase space and can hence be of order $Q$. One thus obtains for the integrated cross sections

$$
\sigma_{\mathrm{SPS}} \sim 1 / Q^{2}, \quad \sigma_{\mathrm{DPS}} \sim \Lambda^{2} / Q^{4},
$$

where DPS has become power suppressed because it populates a smaller phase space. However, DPS can still be important in this case, for instance if SPS is suppressed by coupling constants (the production of $W^{+} W^{+}$or $W^{-} W^{-}$is a prominent example). Generically, DPS is enhanced if the momentum fractions $x$ in the hard scattering subprocesses become small, as already noted in the introduction.

There are further mechanisms that contribute at the same power to the cross section as the terms in (77) or (8), as shown in Ref. 6. In TMD factorisation, the leading power contributions are from SPS, from DPS and from the interference between the two mechanisms. Example graphs are given in Fig. 2(a), (b) and (c).

For the cross section integrated over $\boldsymbol{q}_{1}$ and $\boldsymbol{q}_{2}$, the only leading-power contributions comes from SPS. Suppressed by $\Lambda^{2} / Q^{2}$ are two types of graphs in addition to DPS:

- graphs with a collinear twist-two distribution (i.e. a parton density) for one proton and a collinear twist-four distribution for the other one, as in Fig. 2(d). We refer to this as the twist-four mechanism in the following.

- graphs with a collinear twist-three distribution for each proton, as in Fig. 2(e). This will be referred to as the twist-three mechanism.

As was already noted in Ref. 7 (see also Ref. 4), the integration over $\boldsymbol{q}_{1}$ and $\boldsymbol{q}_{2}$ forces all hard interactions to occur at the same transverse position in the SPS/DPS interference, which thus becomes a special case of the twist-three mechanism. By contrast, in TMD factorisation the graphs in 


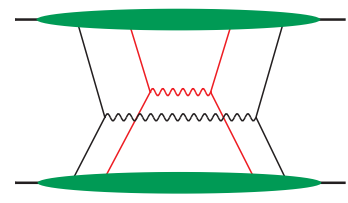

(a)

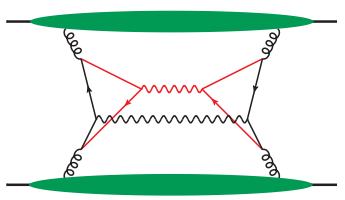

(b)

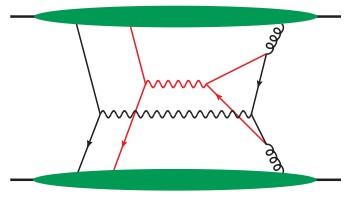

(c)

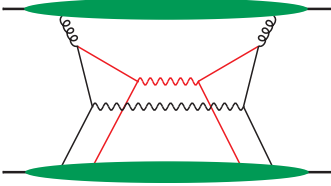

(d)

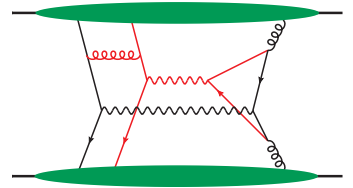

(e)

Fig. 2. Different contributions to the production of two electroweak gauge bosons: DPS (a), SPS (b) and their interference (c). Graphs (d) and (e) involve higher-twist distributions. Internal lines in the hard scattering are off shell by order $Q$. A vertical line for the final state cut is not shown for simplicity.

Fig. 2(d) and (e) are suppressed by $\Lambda^{2} / Q^{2}$ compared with the SPS/DPS interference in Fig. 2(c).

In an unpolarised proton, the number of possible collinear twist-three distributions is severely restricted by helicity conservation, and only distributions with a quark and an antiquark of opposite helicity are allowed $\underline{\underline{8}}$ Such distributions do not have any cross talk with gluon distributions. One can hence expect them to lack the small $x$ enhancement of quark or gluon DPDs, so that there is some justification for neglecting them (in the same spirit as neglecting the parton type interference distributions mentioned in Sec. 21). Notice that in TMD factorisation, the twist-three distributions occurring in the SPS/DPS interference are not subject to restrictions from parton helicity conservation: since all three parton fields are at different transverse positions, orbital angular momentum can compensate a mismatch of parton helicities in this case.

A special class of graphs for the twist-four mechanism, shown in Fig. 3 has been associated with "rescattering" in Ref. 9 (see also Ref. 10). Each propagator marked by a bar in the figure has a denominator of the form $a x-b+i \epsilon$, where $x$ is a loop variable and $a, b$ are fixed by external kinematics. Keeping the pole parts of each propagators and neglecting the principal value part of the integration puts the two lines on shell, and the process looks like one $2 \rightarrow 2$ scattering followed by a second one. The cal- 


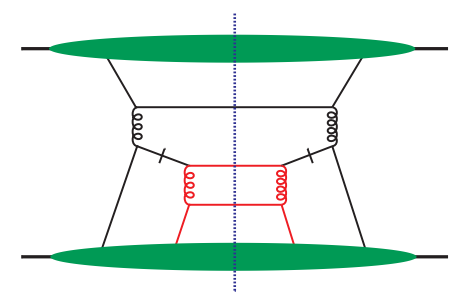

Fig. 3. A graph for three-jet production by the twist-four mechanism that has been associated with "rescattering" in kinematics where the lines marked by a bar are on shell. All three partons in the final state are understood to have large transverse momenta.

culation of this two-pole part in terms of two unpolarised $2 \rightarrow 2$ partonic cross sections is indeed correct if in the twist-four distribution the quantum numbers are coupled such that partons with equal momentum fractions are unpolarised and form a colour singlet. However, it is not obvious that the pole parts of the loop integrations should dominate over the principal value contributions in general kinematics. This may happen for jets with very large rapidities 11 We also emphasise that the partons marked by bars do not physically propagate over distances much larger than $1 / Q$. Technically speaking, their propagator poles can be avoided by a complex contour deformation in the loop integrals, and physically speaking one finds that the "rescattering" of Fig. 3 does not correspond to a classically allowed scattering process. ${ }^{[6]}$ It is therefore inappropriate to associate final- or initial-state parton showers to these partons.

\section{Short-distance splitting and double counting}

At small inter-parton distances, the dominant contribution to a DPD comes from perturbative splitting of one parton into two, as depicted in Fig. 4 (a). Let us for now concentrate on collinear DPDs. At leading order in $\alpha_{s}$, the contribution of the $1 \rightarrow 2$ splitting mechanism is easily computed and reads

$$
\begin{aligned}
& \left.{ }^{R} F_{a_{1} a_{2}}\left(x_{1}, x_{2}, \boldsymbol{y}\right)\right|_{\mathrm{spl}, \mathrm{pt}} \\
& \quad=\frac{1}{y^{2}} \frac{\alpha_{s}}{2 \pi^{2}}{ }^{R} P_{a_{0} \rightarrow a_{1} a_{2}}\left(\frac{x_{1}}{x_{1}+x_{2}}\right) \frac{f_{a_{0}}\left(x_{1}+x_{2}\right)}{x_{1}+x_{2}},
\end{aligned}
$$

where $f_{a_{0}}$ is an unpolarised PDF and $P_{a_{0} \rightarrow a_{1} a_{2}}$ a splitting function. The $1 / y^{2}$ behaviour can be deduced already by dimensional counting. Note that this mechanism gives strong colour and spin correlations: chirality conser- 
vation for massless quarks results for instance in complete anti-alignment of the quark and antiquark helicities in $g \rightarrow q \bar{q}$.

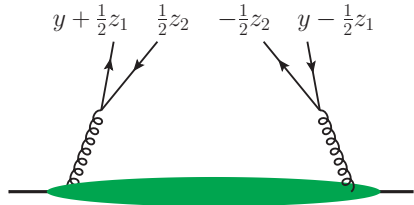

(a)

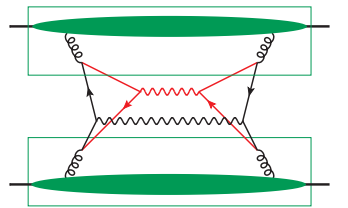

(b)

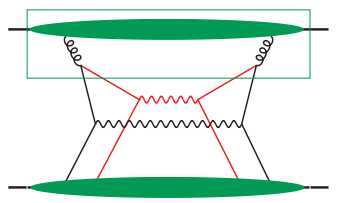

(c)

Fig. 4. Splitting provides a short-distance contribution to DPDs (a) and gives rise to 1v1 (b) and 2v1 (c) graphs for the DPS cross section. The boxes represent double parton distributions; partons emerging from them have virtualities much smaller than $Q$.

DPDs also contain an "intrinsic" short-distance part, where the two partons may be thought of as part of the "intrinsic" wave function of the proton. This part is related to a twist-four distribution and only diverges logarithmically at small $y$. Finally, one may have a $1 \rightarrow 2$ splitting only in the amplitude or only in its conjugate: this contribution involves a collinear twist-three distribution and lacks small $x$ enhancement, as discussed in the previous section.

Inserting the short-distance form (9) into the cross section formula (11), we see that without the function $\Phi$ the integral over $\boldsymbol{y}$ would be power divergent. This power divergence is associated with so-called $1 \mathrm{v} 1$ (1 versus 1 parton) diagrams, in which there are $1 \rightarrow 2$ splittings in both protons as shown in Fig. 4(b). Note that this is the same graph as in Fig. 2(b), which represents a loop correction in the leading-power SPS mechanism. The difference is that in Fig. 四(b) the quark virtualities are understood to be much smaller than $Q$, whereas in Fig. 2(b) they are of order $Q$. The small $y$ divergence in the DPS formula without $\Phi$ is not present in reality: it arises from using DPS approximations in the small $y$ region where they are not valid. It should hence be removed and replaced with the appropriate SPS expression, in a manner that avoids double counting between SPS and DPS. The analogous double counting problem for multi-jet production has already been noticed some time ago.12

A short-distance divergence in the $\boldsymbol{y}$ integral also appears for so-called 2v1 (2 versus 1 parton) diagrams as in figure 4(c), where a $1 \rightarrow 2$ splitting takes place in only one proton. This divergence is only logarithmic, and it corresponds to the overlap of the DPS contribution with the twist-four 
mechanism shown in Fig. 2(d). The importance of the 2v1 mechanism has been emphasised in Refs. 13, 17 ,

In the following we describe a solution to these problems that was elaborated in Ref. 8. Different approaches have been presented in Refs. 13 16 16, the one of Refs. 1314 being reviewed in Ref. 19, A detailed comparison between them is given in Ref. 8. The formalism described here resolves the double counting problem, retains the concept of double parton distributions that have a field theoretic definition, and permits the study of higher-order contributions in a practical way. The other approaches just mentioned do not possess all these features.

The first step is to insert the function $\Phi(y \nu)$ into the cross section formula (11). (We insert the square of $\Phi$ for consistency with the TMD case). This function regulates the divergences just discussed by removing the region $y \ll 1 / \nu$ from what we define to be DPS. It must satisfy $\Phi(u) \rightarrow$ 0 for $u \rightarrow 0$ and $\Phi(u) \rightarrow 1$ for $u \gg 1$. Suitable forms are $\Phi(u)=1-$ $\exp \left(-u^{2} / 4\right)$, or a hard cutoff $\Phi(u)=\Theta\left(u-b_{0}\right)$ with $b_{0}=2 e^{-\gamma_{E}}$ chosen to simplify analytic expressions.

To avoid double counting between DPS and SPS, and between DPS and the twist-four mechanism, we introduce subtraction terms in the overall cross-section:

$$
\sigma_{\mathrm{tot}}=\sigma_{\mathrm{DPS}}-\sigma_{1 \mathrm{v} 1, \mathrm{pt}}+\sigma_{\mathrm{SPS}}-\sigma_{2 \mathrm{v} 1, \mathrm{pt}}+\sigma_{\mathrm{tw} 4} .
$$

The subtraction terms depend on $\nu$ in such a way that the dependence on this unphysical parameter cancels on the right-hand side (to the order of perturbative accuracy of the calculation). Note that $\sigma_{\mathrm{SPS}}$ and $\sigma_{\mathrm{tw} 4}$ do not depend on $\nu$. In particular, $\sigma_{\mathrm{SPS}}$ is simply calculated in the usual way with no modifications. The $1 \mathrm{v} 1$ subtraction $\sigma_{1 \mathrm{v} 1, \mathrm{pt}}$ is constructed in a simple way by replacing the DPDs in the cross section formula (11) by the perturbative splitting approximation (9) or its equivalent at higher orders in $\alpha_{s}$. Similarly, $\sigma_{2 \mathrm{v} 1, \mathrm{pt}}$ is obtained by replacing one of the two DPDs by its splitting approximation and the other one by its intrinsic shortdistance part. There are some subtleties in choosing adequate scales $\mu$ in these distributions, especially if the scales $Q_{1}, Q_{2}, Q_{h}$ of the two DPS subprocesses and of the SPS subprocess are very different, but we will not dwell on this here.

An appropriate choice for the scale $\nu$ is the minimum of $Q_{1}$ and $Q_{2}$. With this choice, $\sigma_{\text {DPS }}$ does contain short-distance contributions for which the DPS approximations are not valid, but these contributions are removed by the subtraction terms in the overall cross section. This is quite similar to 
choosing factorisation scales $\mu \sim Q$ in collinear PDFs and DPDs: the parton distributions then contain virtualities up to the hard scale $Q$, but double counting is avoided by subtractions in the hard-scattering cross sections.

Let us demonstrate how the prescription works. At small $y \sim 1 / Q$, one has $\sigma_{\mathrm{DPS}} \approx \sigma_{1 \mathrm{v} 1, \mathrm{pt}}+\sigma_{2 \mathrm{v} 1, \mathrm{pt}}$ by construction (the product of the intrinsic parts of each DPD gives a power suppressed contribution at small $y$, so that the absence of a subtraction term $\sigma_{2 \mathrm{v} 2 \text {,pt }}$ is no problem). One thus has $\sigma_{\mathrm{tot}} \approx \sigma_{\mathrm{SPS}}+\sigma_{\mathrm{tw} 4}$, as is appropriate for the short-distance region. The dependence on the unphysical cutoff scale $\nu$ cancels between DPS and the subtraction terms. At $y \gg 1 / Q$, the dominant contribution to $\sigma_{\text {SPS }}$ comes from $1 \mathrm{v} 1$ type loops in the region where the DPS approximations are valid, such that $\sigma_{\mathrm{SPS}} \approx \sigma_{1 \mathrm{v} 1, \mathrm{pt}}$. Similarly, we have $\sigma_{\mathrm{tw} 4} \approx \sigma_{2 \mathrm{v} 1, \mathrm{pt}}$. As a result we obtain $\sigma_{\text {tot }} \approx \sigma_{\text {DPS }}$, as appropriate. The construction just explained is a special case of the general subtraction formalism discussed in Chap. 10 of Ref. 1.

For the scale choice $\nu \sim \min \left(Q_{1}, Q_{2}\right)$, one can show that the combination $\sigma_{\mathrm{tw} 4}-\sigma_{2 \mathrm{v} 1, \mathrm{pt}}$ in (10) is subleading compared to $\sigma_{\mathrm{DPD}}$ by a logarithm $\log (Q / \Lambda)$, where $\Lambda$ is an infrared scale. This combination can hence be dropped at leading logarithmic order, which is of great practical benefit since the computation of the twist-four contribution is technically quite involved. For the same scale choice, one finds that $\sigma_{\text {DPD }}$ includes the appropriate resummation of large DGLAP logarithms in the 2v1 graphs.

In order to estimate the theoretical uncertainty from missing higher order terms in this framework, one can vary the parameters $\mu_{1}, \mu_{2}$ and $\nu$, similar to how one varies only $\mu$ in the single scattering case. Note that the variation in $\nu$ of the DPS term alone provides an order-of-magnitude estimate of SPS graphs containing a double box as in Fig. 2(b), since it involves the same PDFs, overall coupling constants and kinematic region (small $y$, corresponding to large transverse momenta and virtualities of internal lines). An alternative estimate is provided by the double counting subtraction term $\sigma_{1 \mathrm{v} 1, \mathrm{pt}}$. Therefore, a small $\nu$ variation of $\sigma_{\mathrm{DPS}}$ compared to its central value indicates that $\sigma_{1 \mathrm{v} 1, \mathrm{pt}}$ and the corresponding loop contribution to $\sigma_{\mathrm{SPS}}$ are negligible compared to $\sigma_{\mathrm{DPS}}$. Several scenarios where the $\nu$ variation is reduced in this way were found in Ref. 8 , for instance when the parton pairs in the relevant DPDs cannot be produced in a single leading-order splitting (e.g. $u \bar{d}$ ), or when low $x$ values are probed in the DPDs. In such cases, one may justifiably neglect the appropriate perturbative order of $\sigma_{\mathrm{SPS}}$, together with the $1 \mathrm{v} 1$ subtraction term. Such processes and kinematic regions are the most promising ones to make useful calcu- 
lations and measurements of DPS, especially because there are only few cases for which SPS is computed at the order containing the double box (essentially only double electroweak gauge boson production).

Now let us turn to the TMD case, where the pattern of overlaps and divergences is somewhat different. In particular, the ultraviolet divergences in $\sigma_{\text {DPS }}$ associated with $1 \mathrm{v} 1$ graphs become logarithmic rather than a power. This is related to the fact that DPS and SPS have the same power behaviour in the small $q_{T}$ region. The inter-parton distance $\boldsymbol{y}_{+}=\boldsymbol{y}+\frac{1}{2}\left(\boldsymbol{z}_{1}-\boldsymbol{z}_{2}\right)$ in the amplitude and its counterpart $\boldsymbol{y}_{-}=\boldsymbol{y}-\frac{1}{2}\left(\boldsymbol{z}_{1}-\boldsymbol{z}_{2}\right)$ in the complex conjugate amplitude (see Fig. 4(a)) are independent variables now. When one of these distances is small, the TMDs are dominated by perturbative $1 \rightarrow 2$ splitting for the corresponding parton pair. The divergent behaviour of $\sigma_{\text {DPS }}$ in the region where both $y_{+}$and $y_{-}$go to zero corresponds to the overlap of DPS with the SPS double box graph in Fig. 2(b). The region

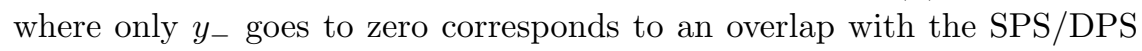
interference graph in Fig. 2(c).

It is clear then that in this case the DPS term must be regulated when either $y_{+}$or $y_{-}$go to zero, as is done in (2). The SPS/DPS interference terms must also be regulated for small $y_{+}$or $y_{-}$, since they overlap with SPS again. Subtraction terms must be included as appropriate to remove the double counting, as elaborated in Ref. 8 ,

\section{Collinear DPDs: evolution}

The twist-two operators in the definition of DPDs contain ultraviolet divergences that require renormalisation. This leads to the familiar DGLAP evolution equations of ordinary PDFs, and to corresponding equations for collinear DPDs. Taking different scales $\mu_{1}, \mu_{2}$ for the partons with momentum fractions $x_{1}$ and $x_{2}$, we have a homogeneous evolution equation

$$
\begin{aligned}
\frac{\partial}{\partial \log \mu_{1}^{2}}{ }^{R} F_{a_{1} a_{2}}\left(x_{1}, x_{2}, \boldsymbol{y} ; \mu_{1}, \mu_{2}, \zeta\right)= & \sum_{b_{1}} \int_{x_{1}}^{1-x_{2}} \frac{d x_{1}^{\prime}}{x_{1}^{\prime}}{ }^{R} P_{a_{1} b_{1}}\left(\frac{x_{1}}{x_{1}^{\prime}} ; \mu_{1}, \frac{x_{1} \zeta}{x_{2}}\right) \\
& \times{ }^{R} F_{b_{1} a_{2}}\left(x_{1}^{\prime}, x_{2}, \boldsymbol{y} ; \mu_{1}, \mu_{2}, \zeta\right)
\end{aligned}
$$

in $\mu_{1}$ and its analogue for $\mu_{2}$. The two parton pairs with momentum fractions $x_{1}$ or $x_{2}$ evolve separately. Note that in the colour singlet sector, both ${ }^{1} F$ and ${ }^{1} P$ are $\zeta$ independent, and ${ }^{1} P$ is the same DGLAP evolution kernel as for ordinary PDFs.

The interplay of DGLAP evolution with the splitting mechanism described in Sec. 4has important consequences when one or both momentum 
fractions $x_{1}, x_{2}$ are small ${ }^{[8]}$ It can change the $1 / y^{2}$ dependence of the splitting contribution (9) into a much flatter $y$ dependence, which increases the contribution of the region $y \gg 1 / \nu$ in the $1 \mathrm{v} 1$ and $2 \mathrm{v} 1$ cross sections. The size of the effect depends on kinematics and on the parton types involved.

The Fourier integral that converts $F\left(x_{i}, \boldsymbol{y}\right)$ into a momentum space DPD $F\left(x_{i}, \boldsymbol{\Delta}\right)$ has a logarithmic divergence at small $y$ from the splitting contribution, which requires additional ultraviolet renormalisation. ${ }^{6}$ In the following, we concentrate on colour singlet distributions $(R=1)$ and on equal scales $\mu_{1}=\mu_{2}$. One way to define $F\left(x_{i}, \boldsymbol{\Delta}\right)$ is to perform the Fourier transform in $D=4-2 \epsilon$ dimensions and use ordinary $\overline{\mathrm{MS}}$ renormalisation for the splitting divergence. The resulting evolution equation has an additional inhomogeneous term, which at $\mathrm{LO}$ in $\alpha_{s}$ reads

$$
\begin{aligned}
& \frac{\partial}{\partial \log \mu^{2}}{ }^{1} F_{a_{1} a_{2}}\left(x_{1}, x_{2}, \Delta ; \mu\right)=\{\text { homogeneous terms }\} \\
& +\frac{\alpha_{s}(\mu)}{2 \pi}{ }^{1} P_{a_{0} \rightarrow a_{1} a_{2}}\left(\frac{x_{1}}{x_{1}+x_{2}}\right) \frac{f_{a_{0}}\left(x_{1}+x_{2} ; \mu\right)}{x_{1}+x_{2}},
\end{aligned}
$$

with the same kernel ${ }^{1} P_{a_{0} \rightarrow a_{1} a_{2}}$ as in (9). The homogeneous terms have the same form as in the evolution of $F\left(x_{i}, \boldsymbol{y}\right)$, with $\boldsymbol{y}$ replaced by $\boldsymbol{\Delta}$. This inhomogeneous evolution has been discussed extensively in the literature ${ }^{20 \mid} 24$

Whilst the scheme presented here requires position space DPDs $F\left(x_{i}, \boldsymbol{y}\right)$ for computing cross sections, the momentum space DPDs have a property that makes their study worthwhile. At $\boldsymbol{\Delta}=\mathbf{0}$, unpolarised momentum space DPDs satisfy sum rules ${ }^{23}$ for the momentum and the flavour quantum numbers of one of the two partons:

$$
\begin{aligned}
\sum_{a_{2}=q, \bar{q}, g} \int_{0}^{1-x_{1}} d x_{2} x_{2}{ }^{1} F_{a_{1} a_{2}}\left(x_{1}, x_{2}, \mathbf{0}\right) & =\left(1-x_{1}\right) f_{a_{1}}\left(x_{1}\right), \\
\int_{0}^{1-x_{1}} d x_{2}\left[{ }^{1} F_{a_{1} q}\left(x_{1}, x_{2}, \mathbf{0}\right)-{ }^{1} F_{a_{1} \bar{q}}\left(x_{1}, x_{2}, \mathbf{0}\right)\right] & =N_{a_{1} q} f_{a_{1}}\left(x_{1}\right),
\end{aligned}
$$

where $N_{a_{1} q}$ is a combinatorial factor. The validity of these sum rules for $\overline{\mathrm{MS}}$ renormalised distributions can be shown to all orders in perturbation theory ${ }^{25}$ A relation between DPDs in momentum and position space can be established by defining distributions

$$
{ }^{1} F_{\Phi}\left(x_{1}, x_{2}, \boldsymbol{\Delta} ; \mu, \nu\right)=\int d^{2} \boldsymbol{y} e^{i \boldsymbol{\Delta} \boldsymbol{y}} \Phi(y \nu){ }^{1} F\left(x_{1}, x_{2}, \boldsymbol{y} ; \mu\right),
$$

where the logarithmic singularity at small $y$ is removed by the same regulator function $\Phi$ used in the cross section. These DPDs and the $\overline{\mathrm{MS}}$ renormalised ones differ only by the treatment of the ultraviolet region, so that 
their difference can be computed in perturbation theory ${ }^{8}$ To order $\alpha_{s}$, one finds that ${ }^{1} F\left(x_{1}, x_{2}, \boldsymbol{\Delta} ; \mu\right)-{ }^{1} F_{\Phi}\left(x_{1}, x_{2}, \boldsymbol{\Delta} ; \mu, \mu\right)$ at $\boldsymbol{\Delta}=\mathbf{0}$ is a calculable function of $x_{1} /\left(x_{1}+x_{2}\right)$ times the inhomogeneous term in (12).

We note that the factorisation formula (1) can be rewritten in terms of $\int d^{2} \boldsymbol{\Delta}{ }^{R} F_{\Phi}\left(\bar{x}_{1}, \bar{x}_{2},-\boldsymbol{\Delta}\right){ }^{R} F_{\Phi}\left(x_{1}, x_{2}, \boldsymbol{\Delta}\right)$. This has been used to show that, at leading logarithmic accuracy, the collinear $2 \mathrm{v} 2$ and $2 \mathrm{v} 1$ cross sections given in Refs. $13-17$ are consistent with the formalism presented here. ${ }^{8}$

\section{Soft gluons and Sudakov logarithms}

The proof of the DPS factorisation formulae (11) and (2) proceeds in close analogy to the case of SPS. Here we only sketch the steps that lead to the construction of DPDs and their evolution equations in rapidity, referring to Refs. 26 and 27 for details. One starts by showing that graphs contributing to the cross section at leading power in $\Lambda / Q$ factorise into hard, collinear and soft subgraphs, as depicted for the double Drell-Yan process in Fig. 5(a). In the hard-scattering subgraphs $H_{1}$ and $H_{2}$ all internal lines are far off shell, the subgraphs $A$ and $B$ involve only momenta collinear to one of the incoming protons, and the subgraph $S$ describes the exchange of soft gluons between the right-moving partons in $A$ and the left-moving ones in $B$.

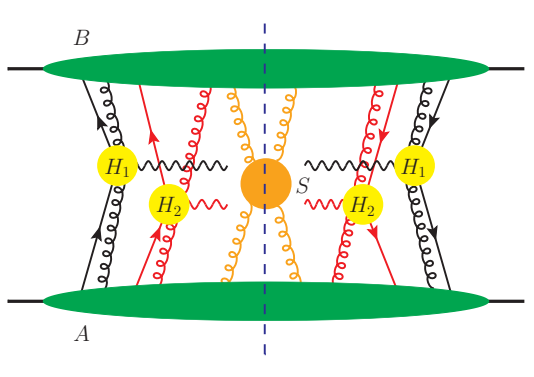

(a)

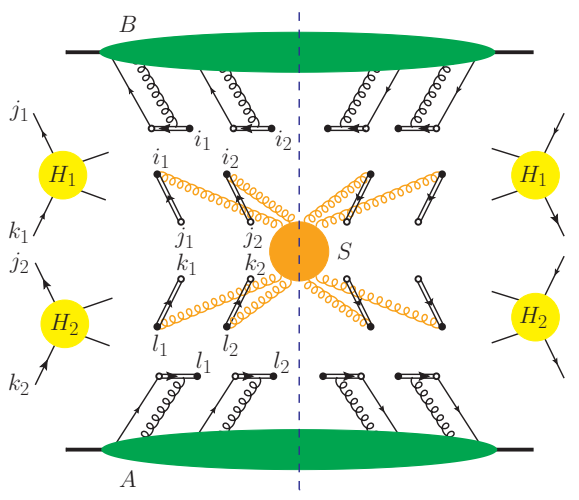

(b)

Fig. 5. (a) Factorised graph for double Drell-Yan production. (b) Graph for double dijet production after the Grammer-Yennie approximation and Ward identities have been applied. $i_{1}, i_{2}, \cdots, l_{2}$ are colour indices. 
To obtain a useful factorisation formula, soft gluons must be decoupled from $A$ and $B$. To achieve this, one performs a Grammer-Yennie approximation. For one gluon with momentum $\ell$ flowing from $S$ into $A$, this reads

$$
S_{\mu}(\ell) A^{\mu}(\ell) \approx S^{-}(\ell) \frac{v_{R}^{+}}{\ell^{-} v_{R}^{+}+i \epsilon} \ell^{-} A^{+}(\tilde{\ell}) \approx S_{\mu}(\ell) \frac{v_{R}^{\mu}}{\ell v_{R}+i \epsilon} \tilde{\ell}_{\nu} A^{\nu}(\tilde{\ell}),
$$

where $v_{R}=\left(v_{R}^{+}, v_{R}^{-}, \mathbf{0}\right)$ specifies a direction with large positive rapidity $Y_{R}=\frac{1}{2} \log \left|v_{R}^{+} / v_{R}^{-}\right|$. In the first step, we have retained only the largest component of $A^{\mu}$ and replaced $\ell=\left(\ell^{+}, \ell^{-}, \ell\right)$ with $\tilde{\ell}=\left(0, \ell^{-}, \ell\right)$ in that factor, which involves plus momenta much larger than $\ell^{+}$. The second step is more delicate and will be discussed in Sec. 7. One can now apply a Ward identity to $\tilde{\ell}_{\nu} A^{\nu}(\tilde{\ell})$, which removes the gluon attachment from $A$. The soft gluon emerging from $S$ then couples to a Wilson line along the direction $v_{R}$. Naively, one would take $v_{R}$ lightlike, i.e. set $v_{R}^{-}=0$, but this would lead to so-called rapidity divergences of loop integrals inside $S$. Instead of a finite rapidity $Y_{R}$, one may use other methods to regulate these divergences, two of which have been applied to DPS in Refs. 28 and 29.

In full analogy, one can replace soft gluons coupling to $B$ by gluons coupling to a Wilson line along a direction $v_{L}$ with large negative rapidity. The soft factor $S$ can now be written as the vacuum expectation value of Wilson line operators and is thus decoupled from $A$ and $B$.

A similar argument is used for longitudinally polarised collinear gluons exchanged between $A$ and $H_{1}$ or $H_{2}$, removing them from the hard scatters and coupling them to Wilson lines along $v_{L}$. These Wilson lines appear in the proton matrix element $\left\langle p\left|\mathcal{O}_{a_{1}} \mathcal{O}_{a_{2}}\right| p\right\rangle$ associated with the factor $A$. The same steps are followed for gluons exchanged between $B$ and $H_{1}$ or $H_{2}$, resulting in Wilson lines along $v_{R}$ in the matrix element associated with $B$.

The factors in the cross section formula are tied together by colour indices in a way that is shown in Fig. 5 (b) for double dijet production. We first discuss the production of colour singlet particles in $H_{1}$ and $H_{2}$. In this case, the soft factor is contracted with $\delta^{j_{1} k_{1}} \delta^{j_{2} k_{2}}$ and analogous factors for the index pairs on the r.h.s. of the final-state cut.

It is useful to project the collinear and soft factors on colour representations $R$, as was explained for DPDs in Sec. 2. The collinear factors then become vectors ${ }^{R} A,{ }^{R} B$ in the space of colour representations, and the soft factor for colour singlet production becomes a matrix ${ }^{R R^{\prime}} S$. In the cross section we have the combination $\sum_{R R^{\prime}}{ }^{R} B R^{\prime} S{ }^{\prime} A$.

The soft matrix in the cross section depends on the rapidity difference $Y=Y_{R}-Y_{L}$ of the Wilson lines along $v_{R}$ and $v_{L}$. This dependence is given 
by a Collins-Soper equation

$$
\frac{\partial}{\partial Y} R R^{\prime \prime} S(Y)=\sum_{R^{\prime}} R R^{\prime} \widehat{K} R^{\prime} R^{\prime \prime} S(Y)
$$

with a rapidity independent matrix $\widehat{K}$. Introducing an intermediate rapidity $Y_{C}$ between $Y_{R}$ and $Y_{L}$, one can write $S(Y)$ as the product of two matrices, one depending on $Y_{R}-Y_{C}$ and the other on $Y_{C}-Y_{L}$. Combining one of them with $A$ and the other with $B$, one obtains DPDs $F_{A}\left(Y_{C}\right)$ and $F_{B}\left(Y_{C}\right)$. In the DPDs, one can take the limits $Y_{L} \rightarrow-\infty$ and $Y_{R} \rightarrow \infty$ without encountering rapidity divergences. The proton matrix element in (4) then contains lightlike Wilson lines. The final cross section formula involves the sum $\sum_{R}{ }^{R} F_{B}{ }^{R} F_{A}$ with colour dependent DPDs, as anticipated in Sec. 2 Note that $A, B$ and $S$ are nonperturbative quantities since they involve low virtualities. Eliminating $S$ by defining distributions $F_{A}$ and $F_{B}$ thus represents a significant simplification.

The construction sketched so far is common to TMD and collinear factorisation. However, ultraviolet renormalisation works differently in the two cases, which we now discuss in turn.

TMD factorisation. It is useful to express the rapidity dependence of the DPDs in terms of boost invariant quantities, $\zeta=2 x_{1} x_{2}\left(p^{+}\right)^{2} e^{-2 Y_{C}}$ for $F_{A}$ and an analogue $\bar{\zeta}$ for $F_{B}$. We concentrate on $F_{A}$ from now on and omit the subscript $A$. Restoring the dependence on all other variables, we have a Collins-Soper equation

$$
\frac{\partial}{\partial \log \zeta}{ }^{R} F\left(x_{i}, \boldsymbol{z}_{i}, \boldsymbol{y} ; \mu_{i}, \zeta\right)=\frac{1}{2} \sum_{R^{\prime}} R R^{\prime} K\left(\boldsymbol{z}_{i}, \boldsymbol{y} ; \mu_{i}\right){ }^{R^{\prime}} F\left(x_{i}, \boldsymbol{z}_{i}, \boldsymbol{y} ; \mu_{i}, \zeta\right)
$$

with a matrix kernel $K$ related to $\widehat{K}$ in (16). Its $\mu_{1}$ dependence is given by

$$
\frac{\partial}{\partial \log \mu_{1}} R R^{\prime} K\left(\boldsymbol{z}_{i}, \boldsymbol{y} ; \mu_{i}\right)=-\delta_{R R^{\prime}} \gamma_{K}\left(\mu_{1}\right)
$$

whilst for the DPD we have

$$
\frac{\partial}{\partial \log \mu_{1}}{ }^{R} F\left(x_{i}, \boldsymbol{z}_{i}, \boldsymbol{y} ; \mu_{i}, \zeta\right)=\gamma_{F}\left(\mu_{1}, x_{1} \zeta / x_{2}\right)^{R} F\left(x_{i}, \boldsymbol{z}_{i}, \boldsymbol{y} ; \mu_{i}, \zeta\right)
$$

with

$$
\gamma_{F}(\mu, \zeta)=\gamma_{F}\left(\mu, \mu^{2}\right)-\frac{1}{2} \gamma_{K}(\mu) \log \frac{\zeta}{\mu^{2}} .
$$

Here $\gamma_{K}(\mu)$ and $\gamma_{F}\left(\mu, \mu^{2}\right)$ depend on $\mu$ via $\alpha_{s}(\mu)$. Analogues of (18) and (19) hold for the $\mu_{2}$ dependence. Note that the kernel $K$ and the anomalous 
dimensions $\gamma_{K}$ and $\gamma_{F}$ differ for quarks and for gluons, but are independent of parton polarisation and quark flavour.

The system of evolution equations can be solved analytically (provided the matrix $K$ can be diagonalised analytically). The solution exponentiates Sudakov double logarithms, controlled by $\gamma_{K}$, and single logarithms going with $\gamma_{F}$ and $K$. Except for the scaling of $\zeta$ by $x_{1} / x_{2}$ or $x_{2} / x_{1}$, the double logarithms have the same form as for single TMDs. When $F_{A}$ is multiplied with $F_{B}$ in the cross section, logarithms of $\zeta$ and $\bar{\zeta}$ turn into logarithms of the invariant masses $Q_{1}$ and $Q_{2}$ in the two hard-scattering subprocesses.

Collinear factorisation. In collinear factorisation, the soft factor simplifies considerably. Using colour algebra, one can show that the general

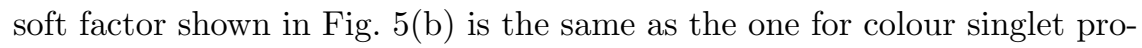
duction, which is contracted with $\delta^{j_{1} k_{1}} \delta^{j_{2} k_{2}}$ etc. Moreover one finds that the soft matrix ${ }^{R R^{\prime}} S$ is diagonal. The colour matrix algebra in the construction of DPDs thus becomes trivial. The product of factors in the cross section can then be written as

$$
\sum_{R}{ }^{R} H_{1}{ }^{R} H_{2}{ }^{R} F_{B}{ }^{R} F_{A} .
$$

If a colour singlet state is produced, then all colour projections ${ }^{R} H$ are equal, otherwise they differ. Multiplying ${ }^{R} H$ with a flux factor, one obtains the subprocess cross sections ${ }^{R} \hat{\sigma}$ in the collinear factorisation formula (1). Collinear DPDs depend on $\zeta$ as

$$
\frac{\partial}{\partial \log \zeta}{ }^{R} F\left(x_{i}, \boldsymbol{y} ; \mu_{i}, \zeta\right)=\frac{1}{2}^{R} J\left(\boldsymbol{y} ; \mu_{i}\right)^{R} F\left(x_{i}, \boldsymbol{y} ; \mu_{i}, \zeta\right)
$$

with

$$
\frac{\partial}{\partial \log \mu_{1}}{ }^{R} J\left(\boldsymbol{y} ; \mu_{i}\right)=-{ }^{R} \gamma_{J}\left(\mu_{1}\right)
$$

and an analogous equation for the $\mu_{2}$ dependence. The DGLAP kernels in the evolution equation (11) depend on $\zeta$ via

$$
{ }^{R} P_{a b}(x ; \mu, \zeta)={ }^{R} P_{a b}\left(x ; \mu, \mu^{2}\right)-\frac{1}{4} \delta_{a b} \delta(1-x){ }^{R} \gamma_{J}(\mu) \log \frac{\zeta}{\mu^{2}} .
$$

The $\zeta$ dependence of the DPDs can be given in analytical form. It contains exponentiated double logarithms controlled by $\gamma_{J}$ and single logarithms going with the kernel $J$. In the colour singlet sector, the soft factor is trivial, ${ }^{11} S=1$, and correspondingly one has ${ }^{1} J={ }^{1} \gamma_{J}=0$. In physical terms, the effects of soft gluon exchange cancel in this case. As a result, colour non-singlet DPDs are suppressed by Sudakov logarithms, whereas colour singlet DPDs are not. ${ }^{28130}$ 


\section{Glauber gluons and factorisation}

A crucial step in showing that soft gluon exchange between the subgraphs $A$ and $B$ in Fig. 5(a) can be subsumed into the vacuum expectation value of Wilson lines is to establish the absence of contributions from the socalled Glauber region. In the following, we restrict ourselves to colour singlet production, since it is the only context in which this issue has been studied. The arguments apply both to collinear and TMD factorisation.

For gluons leaving the soft subgraph $S$ in Fig. 囵(a), there are in fact two distinct momentum regions that contribute to the cross section at leading power. The first one can be called the "central soft" region, where all components of the momentum $\ell$ have comparable size, $\left|\ell^{+}\right| \sim\left|\ell^{-}\right| \sim|\ell|$. In this region, the second step of the Grammer-Yennie approximation (15) is valid: we have $\ell^{-} A^{+} \approx \tilde{\ell}_{\nu} A^{\nu}$ because $\boldsymbol{\ell} \boldsymbol{A}$ is power suppressed compared to $\ell^{-} A^{+}$. The second one is the Glauber region, which is characterised by $\left|\ell^{+} \ell^{-}\right| \ll \ell^{2}$. Gluons in this region mediate small-angle scattering of a right-moving parton on a left-moving one. In the Glauber region, we can have $|\ell \boldsymbol{A}| \sim\left|\ell^{-} A^{+}\right|$, so that the Grammer-Yennie approximation fails. This presents a serious obstacle to factorisation.

Of course, the soft momentum $\ell$ in a graph is not held fixed but integrated over. For many types of soft gluon attachment, the integration over $\ell^{+}$or $\ell^{-}$(or both) can be deformed away from the real axis into the complex plane in such a way that one has $\left|\ell^{+} \ell^{-}\right| \sim \ell^{2}$ on the deformed integration contour and thus avoids the Glauber region. This is only possible when the poles in $\ell^{+}$or $\ell^{-}$of the propagators depending on $\ell$ do not obstruct the deformation. In such cases, the contribution from $\ell$ in the Glauber region can be validly subsumed into the contribution from a collinear or a central soft region, where Grammer-Yennie approximations can be applied to achieve factorisation.

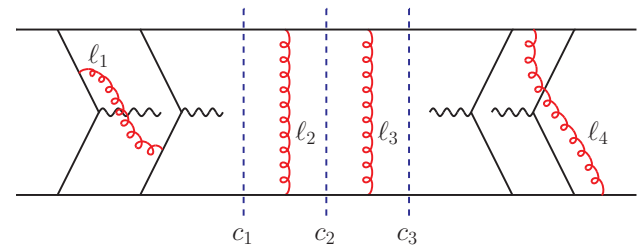

Fig. 6. Graph for double Drell-Yan production with several soft gluons exchanged between left and right moving fast partons. The three possible final state cuts of the graph are denoted by $c_{1}, c_{2}$ and $c_{3}$. 
Examples of soft attachments for which we may deform the momentum out of the Glauber region are given by the gluons with momenta $\ell_{1}$ and $\ell_{4}$ in Fig. 6. In fact, for all of the "novel" types of soft attachment that appear only when we consider DPS rather than SPS, such a deformation in possible. Note that the sign of $i \epsilon$ in the denominators of (15) is chosen precisely such that it does not obstruct these contour deformations. As a consequence, the Wilson lines in the construction of soft factors and DPDs are past pointing (in light-cone coordinates), as in (6).

The one type of soft attachment for which the propagator poles obstruct a deformation out of the Glauber region is exemplified by the gluons with momenta $\ell_{2}$ and $\ell_{3}$ in Fig. 6. This is an attachment between a right-moving and a left-moving spectator parton after the two hard scatters (where "after" refers to the topology of graphs and not to the time coordinate in some reference frame). Of course, such exchanges occur already in SPS. As shown for instance in Ref. 31, the contribution from the Glauber region cancels to leading power when one sums over all final state cuts of a given graph. This requires that the cross section is differential only in properties of the hard scattering products, but fully inclusive over the remaining particles. The same argument applies to DPS. The principle ensuring this cancellation is unitarity: spectator scattering does affect details of the final state, but its net effect is zero if the observable is not sensitive to the spectator momenta. The DPS cross sections (11) and (2) satisfy this requirement. On the other hand, one can show that Glauber gluon exchange does break factorisation for observables depending on the momenta of the spectator partons (or better, of the "beam jets" into which these partons hadronise).

The argument just sketched works for simple graphs, essentially at the level of single soft gluon exchange. To demonstrate Glauber cancellation at all orders, a more powerful technique is needed, based on the light-front ordered version of QCD perturbation theory (LCPT). This argument was given for DPS in Ref. 26, generalising the treatment of the SPS case in Ref. 1. In the LCPT picture, one sees again that from the point of view of the Glauber gluons, single and double hard scattering look rather similar, and that the troublesome "final state" poles obstructing the deformation out of the Glauber region cancel after the sum over of final state cuts. Again, a unitarity argument is used to achieve this cancellation. 


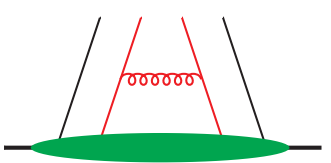

(a)

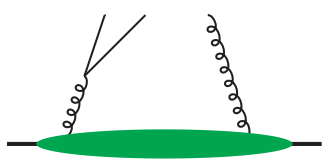

(b)

Fig. 7. (a) Ladder graph for a DPD. (b) Splitting contribution for a twist-three TMD.

\section{Perturbative transverse momenta}

TMD factorisation is applicable when the typical size $q_{T}$ of the transverse momenta $\boldsymbol{q}_{1}, \boldsymbol{q}_{2}$ is much smaller than the hard scale $Q$. This includes the multi-scale regime $\Lambda \ll q_{T} \ll Q$. In this situation, the small parameter in the general power counting of Sec. 3 becomes $q_{T} / Q$ rather than $\Lambda / Q$.

The Fourier exponent $e^{-i\left(\boldsymbol{q}_{1} \boldsymbol{z}_{1}+\boldsymbol{q}_{2} \boldsymbol{z}_{2}\right)}$ in the TMD cross section (2) limits the distances $z_{1}$ and $z_{2}$ to typical size $1 / q_{T}$. Double TMDs can then be computed in terms of perturbative subprocesses at scale $q_{T}$ and of collinear matrix elements expressing the physics at scale $\Lambda$, which significantly increases the predictive power of theory. The distance $y$ is not restricted in this way, and there are in fact two different regimes for DPS.

If $y \sim 1 / \Lambda$ is of hadronic size, then the mechanism generating perturbative transverse momenta is the emission of partons, described by ladder graphs as in Fig. 7(a). For the DPDs we then have

$$
\begin{array}{r}
{ }^{R} F_{a_{1} a_{2}}\left(x_{i}, \boldsymbol{z}_{i}, \boldsymbol{y} ; \mu_{i}, \zeta\right)=\sum_{b_{1} b_{2}}{ }^{R} C_{a_{1} b_{1}}\left(x_{1}^{\prime}, \boldsymbol{z}_{1} ; \mu_{1}, x_{1} \zeta / x_{2}\right) \\
\otimes_{x_{1}}{ }^{R} C_{a_{2} b_{2}}\left(x_{2}^{\prime}, \boldsymbol{z}_{2} ; \mu_{2}, x_{2} \zeta / x_{1}\right) \underset{x_{2}}{\otimes}{ }^{R} F_{b_{1} b_{2}}\left(x_{i}^{\prime}, \boldsymbol{y} ; \mu_{i}, \zeta\right)
\end{array}
$$

with convolution products $\otimes$ as in (11). This is the same mechanism as in SPS (a prominent example is Drell-Yan production with $\Lambda \ll q_{T} \ll Q$ ), and the short-distance coefficients ${ }^{1} C$ are the same as the ones for single TMDs. The relation (25) can be understood in terms of an operator product expansion, with the operators in (5) being expanded for small $z_{1}, z_{2}$ while keeping $y$ large.

The second regime for DPS is when $y$ is of the same size as $z_{i} \sim 1 / q_{T}$. The operator product expansion for the double TMD then involves three types of terms, already presented in Sec. 4. The four partons at small relative distances can originate from a collinear PDF via splitting, as in Fig. 4(a), from a collinear twist-four distribution without any splitting, or from a collinear twist-three distribution with parton splitting only in 
the amplitude or its conjugate. As already noted, the latter case requires chiral-odd distributions and lacks small $x$ enhancement.

In the DPS cross section, we have $1 \mathrm{v} 1,2 \mathrm{v} 1$ and $2 \mathrm{v} 2$ contributions from the four combinations of splitting and intrinsic contributions to the two DPDs. It is important to note that these have different power behaviour in $q_{T}$, namely

$$
\frac{Q^{4} d \sigma}{d^{2} \boldsymbol{q}_{1} d^{2} \boldsymbol{q}_{2}} \underset{y \sim q_{T} \sim 1}{\sim}\left\{\begin{array}{ll}
\alpha_{s}^{2} / q_{T}^{2} & \text { for } 1 \mathrm{v} 1 \\
\alpha_{s} \Lambda^{2} / q_{T}^{4} & \text { for } 2 \mathrm{v} 1 \\
\Lambda^{4} / q_{T}^{6} & \text { for } 2 \mathrm{v} 2
\end{array} \quad \frac{Q^{4} d \sigma}{d^{2} \boldsymbol{q}_{1} d^{2} \boldsymbol{q}_{2}} \underset{y \sim \sim 1}{\sim} \Lambda^{2} / q_{T}^{4},\right.
$$

where we have also specified the behaviour of the contribution from $y \sim$ $1 / \Lambda$. Although this contribution, as well as the $2 \mathrm{v} 1$ part at $y \sim 1 / q_{T}$ are suppressed by $\Lambda^{2} / q_{T}^{2}$ compared with $1 \mathrm{v} 1$, it makes sense to keep them since they have a stronger small $x$ enhancement and involve fewer powers of $\alpha_{s}$. Explicit expressions for the different terms, including the Sudakov factors resulting from $\zeta$ evolution, are given in Ref. 27. We note that (26) holds if $\left|\boldsymbol{q}_{1}+\boldsymbol{q}_{2}\right| \sim\left|\boldsymbol{q}_{1}\right| \sim\left|\boldsymbol{q}_{2}\right|$ are all of order $q_{T}$. Other regimes have been discussed in Refs. 61314.

To obtain the physical cross section, one must combine DPS with SPS and the SPS/DPS interference, as discussed in Sec. 4. The TMDs in these contributions can be expressed in terms of collinear matrix elements as well. For SPS, they are just the ordinary PDFs. For the interference term, one has contributions with collinear twist-three distributions (lacking small $x$ enhancement) and contributions with a PDF and a short-distance splitting only on one side of the final state cut, as shown in Fig. Z(b). Overall, one thus finds that - if collinear twist-three distributions are neglected the only parton distributions needed for TMD factorisation in the regime $\Lambda \ll q_{T} \ll Q$ are collinear DPDs and ordinary PDFs.

\section{Status of factorisation}

Significant progress has been made towards establishing factorisation formulae for DPS processes at the same level of rigour as for SPS. In fact, many of the results we have sketched can even be extended to the case of three or more hard scatterings in a rather straightforward manner. However, a description of the colour structure becomes rather cumbersome in this case, as does the discussion of perturbative splitting and double counting with other mechanisms. To conclude this overview, we list what in our opinion are major remaining open issues in DPS factorisation. 
No all-order proof is available for the nonabelian Ward identities required for decoupling soft gluons from the collinear factors (see Sec. 6). Examples at lowest order have been given in Ref. 6. It may be possible to adapt the proof of the corresponding Ward identities in single Drell-Yan production ${ }^{32}$ to DPS, but this has not been worked out.

A crucial ingredient for constructing DPDs is the evolution equation (16) of the soft matrix $S$, for which no general proof has been given yet. For small distances $y$ and $z_{i}$, one can calculate $S$ in perturbation theory and easily finds that (16) is valid at one loop ${ }^{6}$ Its validity at two loops is corroborated by the calculation in Ref. 29 (which uses a different regulator for rapidity divergences). An all-order proof has recently been put forward in Ref. 33 , but it is currently not clear whether it applies to the rapidity regulator employed in the present work. We also note that the construction sketched below (16) requires $S$ to be positive semidefinite. There is no general proof for this, but it can be motivated by perturbative arguments. ${ }^{27}$

A technical problem in the construction of soft factors are gluons that couple only to Wilson lines along one direction. Such so-called Wilson line self-interactions are divergent for Wilson lines of infinite length. It is easy to see that they cancel in the cross section by construction, but one must also show that they cancel in the individual parton distributions in the factorisation formula. Some discussion for SPS is given in Chap. 13.7 of Ref. 1, but it would be desirable to have a more explicit solution to this problem, before applying it to the case of DPS.

Finally, the cancellation of Glauber gluon exchange has only been shown for DPS processes producing colourless particles. ${ }^{26}$ An extension of this argument to the production of coloured particles, relevant e.g. for jet production, has not even been worked out for SPS, as far as we know. Such an extension may be possible for collinear factorisation, whereas for TMD factorisation there are strong arguments that this cannot even be done for SPS $[$ [3

Many of the subtleties in DPS factorisation, such as the presence of parton correlations and the perturbative splitting mechanism, are by now quite well understood on the theory side. Their phenomenological importance, however, remains to be quantified for many interesting cases. This opens a wide field of studies for the future. 


\section{Acknowledgements}

J.G. acknowledges financial support from the European Community under the FP7 Ideas program QWORK (contract 320389). The figures in this contribution were produced with JaxoDraw

\section{References}

1. J. Collins, Foundations of perturbative QCD. Cambridge University Press, 2013.

2. G. Gustafson and L. Lönnblad, Dipoles in Impact Parameter Space and rapidity, contribution to this book.

3. T. C. Rogers and P. J. Mulders, No Generalized TMD-Factorization in Hadro-Production of High Transverse Momentum Hadrons, Phys. Rev. D81 (2010) 094006, 1001.2977.

4. G. Calucci and D. Treleani, Multiple Parton Interactions, inclusive and exclusive cross sections, sum rules, contribution to this book, [1707.00271.

5. T. Kasemets and S. Scopetta, Parton correlations in double parton scattering, contribution to this book.

6. M. Diehl, D. Ostermeier and A. Schäfer, Elements of a theory for multiparton interactions in QCD, JHEP 03 (2012) 089, [111.0910.

7. G. Calucci and D. Treleani, Incoherence and Multiple Parton Interactions, Phys. Rev. D80 (2009) 054025, 0907.4772.

8. M. Diehl, J. R. Gaunt and K. Schönwald, Double hard scattering without double counting, JHEP 06 (2017) 083 , 1702.06486.

9. N. Paver and D. Treleani, Multiple parton processes in the TeV region, $Z$. Phys. C28 (1985) 187.

10. T. Sjöstrand, The Development of MPI Modelling in PYTHIA, contribution to this book, 1706.02166 .

11. D. Treleani, AGK cutting rules and perturbative QCD, Int. J. Mod. Phys. A11 (1996) 613-654.

12. M. Cacciari, G. P. Salam and S. Sapeta, On the characterisation of the underlying event, JHEP 04 (2010) 065, 0912.4926.

13. B. Blok, Yu. Dokshitser, L. Frankfurt and M. Strikman, $p Q C D$ physics of multiparton interactions, Eur. Phys. J. C72 (2012) 1963, 1106.5533.

14. B. Blok, Yu. Dokshitzer, L. Frankfurt and M. Strikman, Perturbative QCD correlations in multi-parton collisions, Eur. Phys. J. C74 (2014) 2926. 1306.3763 .

15. M. G. Ryskin and A. M. Snigirev, A Fresh look at double parton scattering, Phys. Rev. D83 (2011) 114047, 1103.3495.

16. M. G. Ryskin and A. M. Snigirev, Double parton scattering in double logarithm approximation of perturbative QCD, Phys. Rev. D86 (2012) 014018, 1203.2330.

17. J. R. Gaunt, Single Perturbative Splitting Diagrams in Double Parton Scattering, JHEP 01 (2013) 042, 1207.0480. 
18. A. V. Manohar and W. J. Waalewijn, What is Double Parton Scattering?, Phys. Lett. B713 (2012) 196-201, 1202.5034.

19. B. Blok and M. Strikman, Multiparton pp and $p A$ collisions - from geometry to parton-parton correlations, contribution to this book, 1709.00334.

20. R. Kirschner, Generalized Lipatov-Altarelli-Parisi Equations and Jet Calculus Rules, Phys. Lett. B84 (1979) 266-270.

21. V. P. Shelest, A. M. Snigirev and G. M. Zinovev, The Multiparton Distribution Equations in QCD, Phys. Lett. B113 (1982) 325

22. A. M. Snigirev, Double parton distributions in the leading logarithm approximation of perturbative QCD, Phys. Rev. D68 (2003) 114012 , hep-ph/0304172.

23. J. R. Gaunt and W. J. Stirling, Double Parton Distributions Incorporating Perturbative QCD Evolution and Momentum and Quark Number Sum Rules, JHEP 03 (2010) 005, 0910.4347.

24. F. A. Ceccopieri, An update on the evolution of double parton distributions, Phys. Lett. B697 (2011) 482-487, 1011.6586.

25. P. Plößl, DPD sum rules in QCD, in Proceedings, 8th International Workshop on Multiple Partonic Interactions at the LHC (MPI@LHC 2016): San Cristóbal de las Casas, Mexico, November 28-December 2, 2016, 2017, 1703.10875 .

26. M. Diehl, J. R. Gaunt, D. Ostermeier, P. Plößl and A. Schäfer, Cancellation of Glauber gluon exchange in the double Drell-Yan process, JHEP 01 (2016) $076,1510.08696$.

27. M. G. A. Buffing, M. Diehl and T. Kasemets, Transverse momentum in double parton scattering: factorisation, evolution and matching, 1708.03528

28. A. V. Manohar and W. J. Waalewijn, A QCD Analysis of Double Parton Scattering: Color Correlations, Interference Effects and Evolution, Phys. Rev. D85 (2012) 114009, 1202.3794.

29. A. Vladimirov, Soft factors for double parton scattering at NNLO, JHEP 12 (2016) 038, 1608.04920.

30. M. Mekhfi and X. Artru, Sudakov Suppression of Color Correlations in Multiparton Scattering, Phys. Rev. D37 (1988) 2618-2622

31. J. R. Gaunt, Glauber Gluons and Multiple Parton Interactions, JHEP 07 (2014) 110, 1405.2080

32. J. C. Collins, D. E. Soper and G. F. Sterman, Soft Gluons and Factorization, Nucl. Phys. B308 (1988) 833-856

33. A. Vladimirov, Structure of rapidity divergences in soft factors, 1707.07606 .

34. D. Binosi, J. Collins, C. Kaufhold and L. Theussl, JaxoDraw: A Graphical user interface for drawing Feynman diagrams. Version 2.0 release notes, Comput. Phys. Commun. 180 (2009) 1709-1715, 0811.4113. 